\title{
NUEVOS DOCUMENTOS EPIGRÁFICOS DEL VALLE DEL DUERO: UN ALTAR DEDICADO A PLUTÓN Y UNA ESTELA FUNERARIA RUBRICATA
}

\author{
POR \\ CARMEN GARCÍA MERINO \\ Universidad de Valladolid
}

\section{RESUMEN}

Se presenta un ara votiva consagrada al dios infernal Plutón como Dis Pater, hallada al oeste de la provincia de Soria entre las ciudades de Clunia y Uxama, elocuente documento de un culto apenas atestiguado epigráficamente en Hispania. También se da a conocer una pequeña estela que conserva evidencias claras de rubricatio y es, por ahora, la única inscripción relacionable con la villa romana de Almenara de Adaja (Valladolid)

\section{SUMMARY}

This note presents two epigraphic documents. One is a votive altar consecrated as Dis Pater to the hell god Pluto. This was found in the West part of Soria province, between the towns of Clunia and Uxama, and affords an eloquent example from a cult scarcely documented in the epigraphy of Hispania. The other is an small funerary limestone with still keeps evidences of rubricatio. Up to now this is the only inscription related to the Roman villa at Almenara de Adaja (Valladolid).

PALABRAS CLAVE: Documentación epigráfica. Culto a Dis Pater. Inscripción funeraria. Coloración del campo epigráfico.

KEY WORDS: Epigraphic documents. Dis Pater's Cult. Funerary inscription. Rubricatio.

\section{ORANDO A PLUTÓN: EL ALTAR DE GUI- JOSA (SORIA) DEDICADO A DIS PATER}

Se trata de un altar de caliza hecho de una pieza. Sus dimensiones son $32 \mathrm{~cm}$ de altura conservada, 13,3 $\mathrm{cm}$ de anchura y 12,3 de grosor. El coronamiento, roto en la base y los laterales, tiene $7 \mathrm{~cm}$ de altura, carece de pulvini y está moldurado con un toro y media escocia. Cuatro depresiones de forma triangular con lados redondeados de $3 \times 5 \mathrm{~cm}$ bordean un foculus de $6 \mathrm{~cm}$ de diámetro semejando una cuatripétala (Fig. 1). La pieza se encuentra parcialmente rota en la parte baja por tres de sus lados y en la superior por ambos costados. La cara mejor conservada es el dorso.
El altar fue recogido por un particular en el despoblado de Cañicera, en término de Guijosa, Ayuntamiento de Espeja de San Marcelino (Soria), entre las ruinas de la ermita de San Juan Bautista de uno de cuyos lienzos había formado parte. En 2006 se entregó para su traslado al Museo Provincial (Museo Numantino) donde se encuentra en la actualidad ${ }^{1}$. En cuanto al contexto local, hay en las inmediaciones de la ermita un yacimiento romano, el Ortigal, ya señalado por Taracena (Idem 1941, 85) y prospectado nuevamente en 1998 y $1999^{2}$. Se encuentra en una zona llana de la margen derecha del río Pilde donde en superficie se observan materiales de construcción (destacan fustes de columna) y cerámicos, en función de los cuales los prospectores lo clasifican como asentamiento rural/villa de época altoimperial de 2,52 has de extensión ${ }^{3}$. Aunque en el entorno de Cañicera hay a pocos $\mathrm{km}$ de distancia varios yacimientos rurales de cronología imperial ${ }^{4}$, parece lógico señalar como candidato probable al yacimiento del Ortigal por ser la cantera de ruinas reaprovechables mas cercana.

El campo epigráfico ocupa dos tercios de la cara anterior del neto sin delimitación y su estado de con-

\footnotetext{
${ }^{1}$ Agradecemos a los arqueólogos R. Garnelo y A. Balado de la empresa Unoveinte, a quienes el hallador entregó la pieza, su generoso ofrecimiento para estudiarla.

${ }^{2}$ Las prospecciones se realizaron en 1998 y 99 por la empresa Arquetipo S. L. Informe depositado en el Servicio Territorial de Cultura de Soria.

${ }^{3}$ Las coordenadas del yacimiento son $41^{\circ} 45^{\prime} 33^{\prime}-3^{\circ}$ $15^{\prime} 11^{\prime \prime}$. Su referencia es 42.080-0002-03 del Inventario arqueológico de la provincia de Soria existente en el Servicio Territorial de Cultura de Soria que se basa en la ficha del Ortigal debida a M. Lerín, de la empresa Arquetipo (Informe citado en nota 2).

${ }^{4}$ En Espeja de San Marcelino, Hinojosa, Orillares, Quintanilla de Nuño Pedro, Fuentearmegil y Zayuelas, todos del Ayuntamiento de Fuentearmegil, hay yacimientos de hábitat de época imperial. Muy cercanos están también el establecimiento de Alcubilla de Avellaneda, próximo a Clunia, y la villa de Los Villares (Santervás del Burgo) en territorio uxamense.
} 


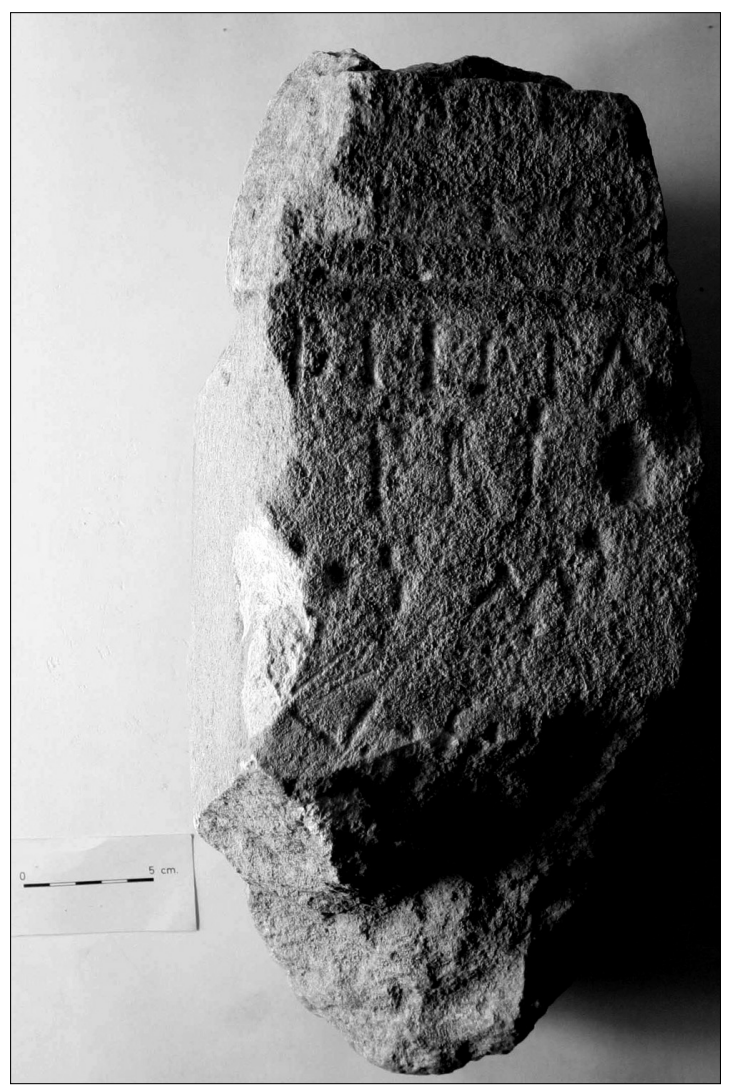

Fig. 1. Altar dedicado a Dis Pater de Guijosa (Soria). Fotografía J .C. Barrena.

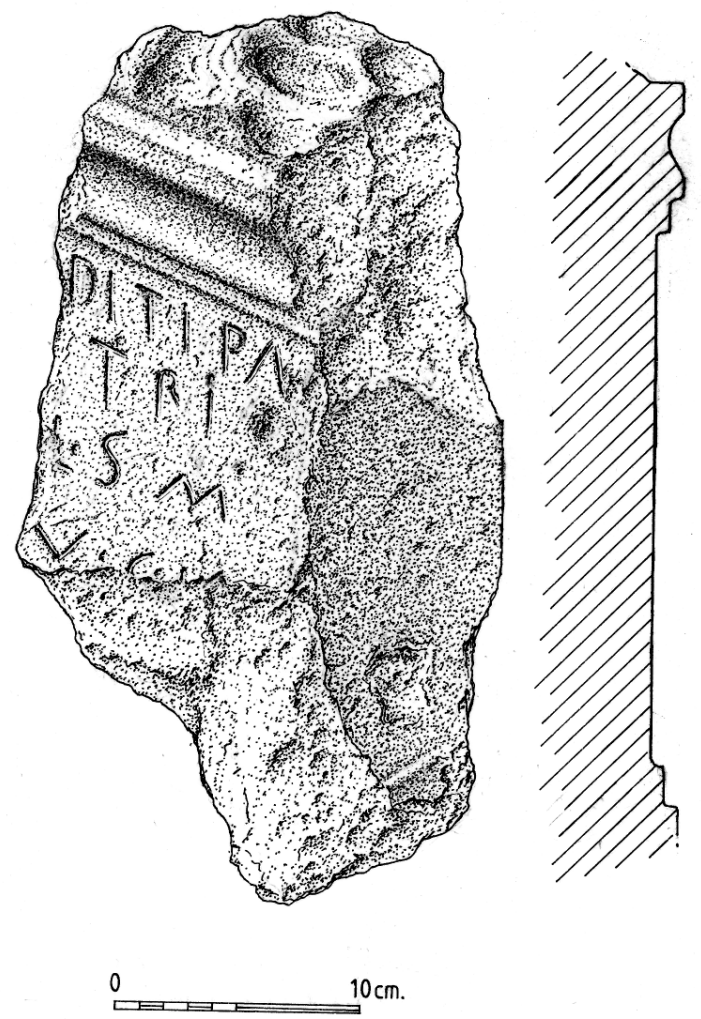

Fig. 2. Altar de Guijosa (Soria). Dibujo de A. Rodríguez. Obsérvese el coronamiento.

lación con el propio culto, o un posible carácter doméstico que obviaría la pretensión de dejar claro quién hizo la ofrenda.

El aparente arcaísmo de la advocación de HadesPlutón como Dis Pater no es base suficiente para fechar el ara ya que se trata de un culto cuya trayectoria en Hispania se desconoce, lo que invalidaría su empleo como referente temporal. Por otra parte, podría tratarse de una vertiente popular del culto, más difícil aún de datar.

La escritura es en letra capital a surco alargada y de factura irregular, mas cuidada en las dos primeras líneas donde figura el nombre del dios; presenta signos de interpunción circulares. El ductus es poco profundo, el ojo de la $\mathrm{P}$ abierto y la $\mathrm{R}$ mal trazada (su ojo apenas está cerrado y el trazo oblicuo es muy corto). Las medidas correspondientes a los elementos de la ordinatio son:

Alt. mín 1.: 1,5. Alt. máx. 1. 2,3. Alt. media interl.: 1. Línea 1: 1,5. Línea 2: 2,3. Línea 3: 2. Línea 4: 2,3. Interl. 1: 0,8. Interl. 2: 0,9. Interl. 3: 2,5. 


\author{
Diti Pa \\ tri \\ L(ucius) $S(-$ ?) $M(-$ ?) \\ $v$ (otum) s(olvit) l(ibens) [a(nimo) $/ \mathrm{m}($ erito $) ?]$
}

Aparato crítico: $\mathrm{El}$ trazo horizontal de las $\mathrm{T}$ de la primera y de la segunda líneas está deformado por un ligero surco curvo, arañado seguramente en tiempos recientes (Fig. 2). La R de la segunda línea está muy mal grabada, marcando poco el ojo, a pesar de ello la lectura no ofrece dudas.

En la línea 3 que consta de tres siglas extendidas, separadas entre sí $2 \mathrm{~cm}$ y con interpunción, la primera letra es seguramente una L. En la cuarta línea, muy estropeada, que constaba de cuatro caracteres ocupando toda la anchura del campo, sólo el primero, $\mathrm{V}$, es perceptible en su totalidad. Luego hay un signo de interpunción y, a continuación, la parte superior de una $\mathrm{S}$, otro signo circular, la parte superior de L y un trazo angular que podría corresponder tanto a una A como a una $\mathrm{M}$, es decir, tanto a a(nimo) como a $m($ erito). En cualquier caso es claro que esta línea alude a la razón de la dedicatoria. Se omite la fórmula habitual de consagración y resulta verosímil que las tres siglas de la tercera línea indiquen el nombre del dedicante. Tal economía gráfica podría deberse en principio a la escasez de espacio disponible.

Nos inclinamos por la restitución S(empronius) del nomen del dedicante debido a la cercanía del lugar del hallazgo del altar a Clunia en varios de cuyos epígrafes aparece esta gens. De hecho, es el segundo gentilicio mas frecuente de los que se citan en ellos, más aún que Pompeius, siendo sólo superado por Valerius (Palol y Vilella,1987, 170). Por su parte, la tercera sigla proponemos desarrollarla como M(aternus) por ser este cognomen muy frecuente en general, y porque en Clunia otros de carácter similar, como Fraternus o Paternus/a, se asocian también con Sempronius/Sempronia (Palol y Vilella, ibidem). Si bien no se pueden descartar otros asimismo relativamente abundantes, como $M$ (artialis), por ejemplo.

(Consagrado) a Dis Pater. Lucio S(empronio?) M(aterno?) cumplió el voto gustosamente.

Comentario: La extraña forma del coronamiento, para la que no conocemos paralelo, con cuatro depresiones en torno al foculus, no sabemos interpretarla; tal vez tenga que ver con algún detalle del rito, por ejemplo, ofrendas para otras cuatro entidades, además del propio Plutón, de aquellas que, como Proserpina y Hécate entre otros, le acompañan en las fórmulas mágicas en cuanto señores del mundo de ultratumba.
En Hispania solo se pueden citar otros dos documentos epigráficos de culto a Plutón, ambos en su advocación de Dis Pater y ambos de procedencia meridional. Uno de ellos, descubierto en 1885 en una casa de Burguillos del Cerro (Badajoz) sin que se conozca su procedencia concreta, se guarda en la Casa de Pilatos (Sevilla). Es la mitad inferior de un ara de mármol gris que sólo conserva cuatro líneas de su texto original, muy fragmentadas las dos primeras. En la última línea: ...../[-]Ma[-]/Zetonia+[-3?-]/ Domino Dit[i] Patri. a(nimo). 1(ibens).v(otum). s(olvit). (EE IX 141=ERBC 56=HEp 7, 1997, 51, Gimeno y Stylow, 2004, 79-80, fig.2, AE, 2004, 739 y HEp 13, 87).

La segunda inscripción, conservada en el Museo Arqueológico Provincial de Sevilla, procede de Munigua (Villanueva del Río y Minas, Sevilla). Pertenece a un pedestal de granito (AE 1975, 501 y 1980, 561. González 1996, 577-58, n. o 1056, fig. 625 y HEp $7,915)$ que deja memoria de la ofrenda de una escultura equina que seguramente lo coronó, al Numen de Dis Pater: Num[ini/]/Ditis. P[atris]/L(ucius). Aelius . Q]uir(ina tribu)/Front[o]/equum . exed[ram]/ equilem (sic). dedit. Nada sorprendente en cuanto a lo dedicado a esta divinidad a la que con frecuencia se representa en un carro tirado por cuatro caballos (negros) (Linder 1988, 213) y a la que se sacrificaban équidos de ese color, si bien lo raro es que el caballo vaya acompañado de establo (Grünhagen 1975, 201-208). Lástima que la lectura que A. Canto propone de las líneas 5 y 6 : equum ex pl(umbeo?/ equile m(armoreum), (HEp 7, 915) no esté justificada por el propio texto, por mucho que pueda parecer idónea la mención de ese metal, ya que el plomo es la materia relacionada con el reino de los muertos y la práctica de la magia (pequeña plástica representando cabras, por ejemplo) y también el soporte de los textos de defixio dirigidos a las divinidades del inframundo gobernado por Plutón. En cuanto al dedicante, L. Aelius Fronto, también expresa su nombre con el sistema triple, posiblemente se trate de un epígrafe de cronología similar.

El lugar de procedencia de la pieza que nos ocupa se enmarca en una zona situada entre Uxama y Clunia, más cerca de esta última, a cuyo territorio probablemente pertenecería. Que el altar se erigiese en la zona arévaca romanizada no sería argumento suficiente para considerar esta dedicación fruto de la interpretatio romana de una divinidad indígena, Tarannis o Endovellico, con Plutón como se ha propuesto (Blázquez 1981, 192 y fig. 33; Id, 1983, 184). A las ciudades mencionadas, particularmente a la segunda por su carácter de capital de conventus, cabe su- 
ponerles una población cosmopolita y la práctica de cultos importados, como el de Neptuno (Palol y Vilella 1987, 31, no 19). Recordemos en este sentido también la imagen de Saturno, divinidad poco habitual, hallada en la cercana villa de los Quintanares de Rioseco (Balil 1984, 325-340) en el territorio probable de Uxama. Se trata de la copia romana altoimperial de un original helenístico, venerada seguramente en una prolongada práctica doméstica del culto todavía en el Bajo Imperio en lo que debió ser una espléndida residencia rural.

\section{INSCRIPCIÓN FUNERARIA RUBRICATA DE ALMENARA DE ADAJA (VALLADOLID)}

Éste es el primer documento epigráfico que se conoce del conjunto arqueológico de La Calzadilla en Almenara de Adaja. El soporte de caliza yesosa, de torpe factura, podría describirse tanto como una estela funeraria con remate triangular entre acróteras (con indicios de un relieve circular, tal vez esbozo de símbolos astrales), extraña por sus reducidas dimensiones, como un arula funeraria. El dorso y los costados están toscamente desbastados (Fig. 4). Le falta la parte inferior. Fue encontrada al labrar las tierras que rodean la zona residencial excavada de la villa romana de La Calzadilla, situada entre Almenara de Adaja y Puras (Valladolid) ${ }^{5}$. Quienes la recogieron no han precisado la fecha del hallazgo. Se encuentra temporalmente en el Museo de las villas romanas de Almenara de Adaja (Valladolid), desde donde pasará al Museo Arqueológico Provincial. Las dimensiones de la estela son: $28 \mathrm{~cm}$ altura máxima conservada, $14,5 \mathrm{~cm}$ de anchura y $8,7 \mathrm{~cm}$ de grosor. Su estado de conservación es deficiente pues está rota en la parte inferior y erosionada en la cartela del texto.

El campo epigráfico de 9 × $9 \mathrm{~cm}$ de superficie está centrado en la mitad superior de la pieza. Es un recuadro rehundido con $3 \mathrm{~mm}$ de profundidad que contiene cuatro líneas de texto. Fuera de él y debajo hay una quinta línea. El cuadrante superior derecho está casi perdido y sólo se percibe parte de algunos trazos. En cuanto a la paleografía, la letra es capital rústica trazada a surco de $3 \mathrm{~mm}$ de profundidad en las tres primeras líneas y menos en la cuarta y quinta. Es irregular y mal ejecutada pero el surco está pintado en rojo con minio. A pesar de la

\footnotetext{
${ }^{5}$ Agradecemos al Sr. Arroyo, quien la descubrió, su entrega con destino al Museo Provincial y a M. Sánchez Simón, codirectora de las excavaciones de la villa de Almenara, las gestiones para recuperarla y dárnosla a conocer.
}

rubricatio la lectura es difícil por la erosión de la superficie debido, sobre todo, a la mala calidad de la piedra.

La ordinatio presenta el texto distribuido en cinco líneas con caracteres mayores en la primera que en las restantes. A este respecto las dimensiones son: Alt. mín. de l.: 1,3. Alt. máx. de l.: 2,2. Alt. media interl.: 0,5. Línea 1/2: 2,2. Línea 3: 1,3. Línea 4/5: 1,5. Interl. 1: 1/2: 0,5. Interl. 3: 0,3. Interl. 4: 1,5.

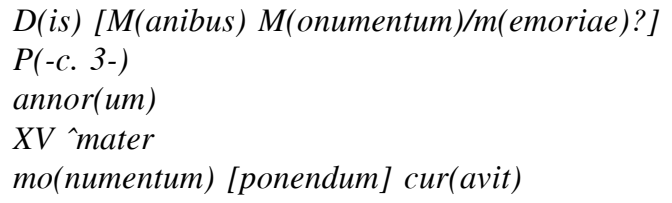

Aparato crítico: La primera línea constaba de tres letras pero sólo se ha conservado la inicial que lleva a la restitución de la siguiente como $\mathrm{M}$ y, aunque la tercera podría ser la recurrente $S$ de la consagración, consideramos más apropiada por la estructura del texto $\mathrm{M}$ (onumentum) o $\mathrm{M}$ (emoriae) como en una estela de procedencia muy cercana de la provincia de Valladolid (Mañanes y Solana, 1999, 25, n. ${ }^{\circ} 11$ y Lám. I). Teniendo en cuenta que más adelante hay otra mención a monumentum, podría ser preferible memoriae, que, bien es verdad, no tiene en la zona paralelos. En la segunda línea, muy destruida como la anterior por una depresión circular tal vez debida a un golpe, son perceptibles la primera letra, una $\mathrm{P}$, y algún pequeño trazo de otros tres signos. De todos modos, parece claro que contenía el nombre del difunto/a. En la tercera línea los caracteres tercero y quinto incompletos, corresponden a $\mathrm{N}$ y $\mathrm{R}$ respectivamente de la expresión de los años. En la cuarta línea tras el numeral de la edad del difunto hay un nexo MA seguido de lo que entendemos como una $\mathrm{T}$ de cuyo brazo horizontal, muy someramente grabado y casi borrado por la erosión de la superficie, solo es perceptible el extremo derecho. Aunque en la quinta línea, fuera de la cartela sólo cuatro de los seis caracteres que la componen se ven medianamente bien: mocur (fig. 3), nos inclinamos por restituir la fórmula mo(numentum) cur(avit), con el empleo ciertamente raro de curare - con lo que hay que suponer elidido ponendum - en lugar del más directo ponere.

Así pues la inscripción ofrece en su primera línea, aparte de la más usual $D$ (iis) [M(anibus sacrum)], otras dos posibles lecturas:

a) $D$ (iis) [M(anibus M(onumentum)

b) $\mathrm{D}$ (iis) $[\mathrm{M}$ (anibus $\mathrm{M}$ (emoriae) 


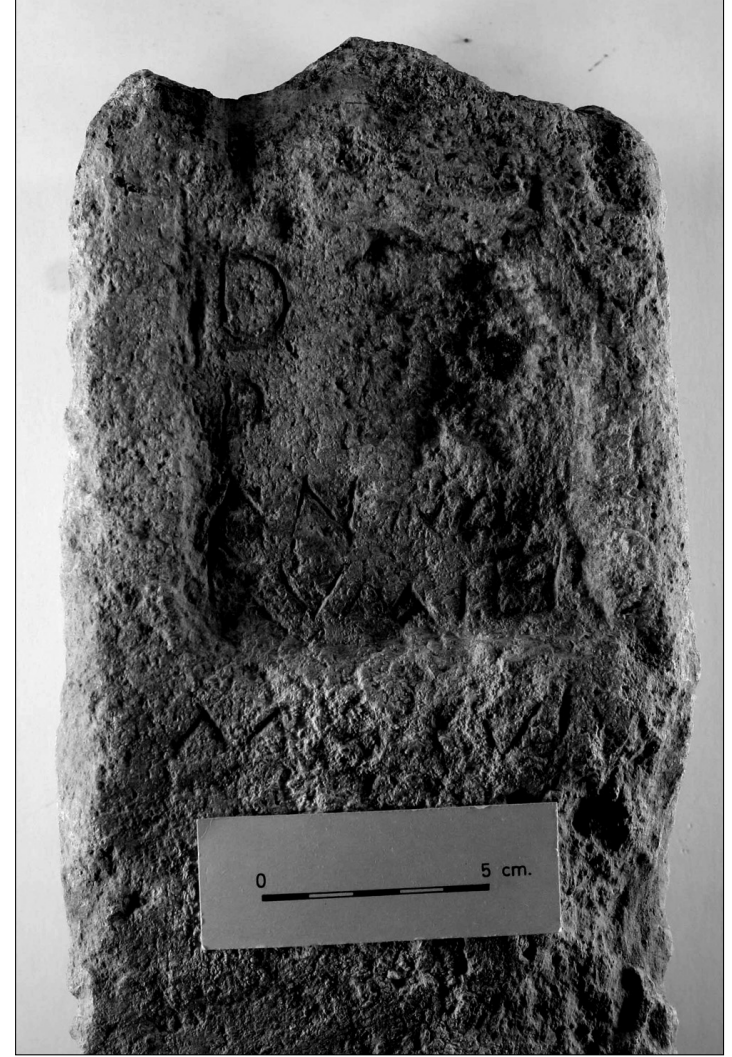

Fig. 3. Inscripción de Almenara de Adaja (Valladolid). Fotografía J. C. Barrena.

En función de lo dicho:

a) (Consagrado) a los Manes. Monumento de $P$---, de XV años. Su madre se encargó (de que se pusiera) el monumento

b) ( Consagrado) a los Manes. A la memoria de $\mathrm{P}$-, de XV años. Su madre se encargó (de que se pusiera) el monumento

Cronología: siglos III-IV Justificación: La fórmula de la edad con la expresión annor(um), poco habitual, se puede poner en relación más con la forma tardía annorum, que con ann(orum). Asimismo, la expresión monumentum posuit como en este caso monumentum curavit corresponde a una fecha avanzada, siglo IV, incluso v, a pesar de sus fórmulas paganas. La tosquedad de la realización de la estela de Almenara, evoca más bien una fase en que los talleres epigráficos urbanos estarían en decadencia y para inscripciones privadas humildes se recurriría a artesanos de la piedra cuya impericia epigráfica queda patente. Por otro lado, su hallazgo en las inmediaciones de una villa tardo-romana precedida por otra

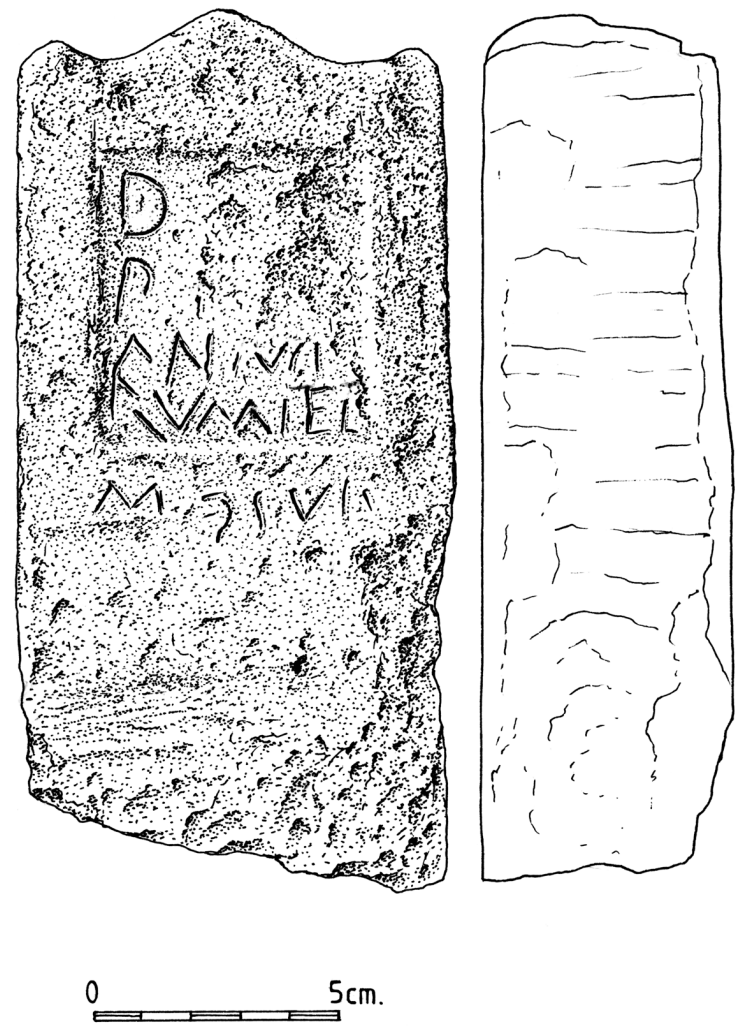

Fig. 4. Inscripción de Almenara de Adaja (Valladolid). Dibujo de A. González.

instalación del siglo III, y considerando que las necrópolis asociadas a villas no son generalmente altoimperiales, parece reforzar la baja cronología apuntada. Reconocemos que no son argumentos concluyentes sino indicios, pero todos apuntan en la misma dirección. La estela de Almenara podría ser, como la cercana de Roda de Eresma (Molinero 1971, 67, lám. CLXI, fig. 2; Knapp 1982, n. ${ }^{\circ} 288$ ), un exponente de la práctica epigráfica pagana aún a finales del siglo III o en el siglo IV en el medio rural del valle del Duero, como ocurría en territorio cántabro según ha demostrado Abascal en un interesante trabajo (Abascal 2002, passim y 290).

Comentario: Es de destacar en la inscripción de Almenara la conservación de la rubricatio. La permanencia del color apunta a que el epitafio no estuvo expuesto a la intemperie, sino a cubierto, tal vez en una construcción funeraria. Se observa en el campo epigráfico que la pigmentación roja no se limita al surco de las letras sino que aparece también en su interior (Fig. 3). Se aprecia sobre todo en las líneas 3 y 4 que han conservado mejor la coloración que las 
dos anteriores por estar protegidas en el rehundido del campo epigráfico. Por ello hay que pensar que tanto la cartela como la zona que inmediatamente debajo cobija la quinta línea de texto, estuvieron coloreadas. La pintura roja sobre el fondo claro de la piedra no solo facilitaba la lectura, sino que, además, compensaba la torpe ejecución de la pieza, propia de una artesanía rudimentaria. En este caso el pigmento empleado es el minio (tetróxido de plomo, $\mathrm{Pb}_{3} \mathrm{O}_{4}$ ), utilizado normalmente en la rubricatura de epígrafes y en la pintura mural. No se debe confundir con el cinabrio (sulfuro de mercurio, $\mathrm{HgS}$ ), mucho más caro, que se restringía a un grupo muy selecto de inscripciones sobre mármol y a decoraciones pictóricas de calidad excepcional. La denominación indiscriminada de cinabrio a ambos pigmentos se gestó a partir del propio texto de Plinio donde se menciona esa aplicación del rojo -cinaber- a la escritura sobre piedra (Plin. nat. 33, 122, H. Rackham, Loeb 1952) y ha durado hasta el siglo xVIII.

Aunque el repaso con pintura roja fue práctica frecuente para resaltar las letras, o en casos de inscripciones de mala calidad para facilitar la lectura de un texto someramente inciso, o incluso, para escribir sin grabar previamente como ya se ha señalado (Abascal 2002, 285), lo que resulta infrecuente es la conservación del color que se da muy pocas veces. La exposición a la intemperie en unos casos y la acidez del suelo en otros han determinado que dispongamos de muy pocas evidencias, especialmente en soportes de piedra blanda o muy sensible a la erosión. Se pueden citar algunos otros casos de textos con indicios de esa práctica, por ejemplo en la meseta, dos inscripciones funerarias de Segovia, reutilizadas en la muralla y guardadas en el Museo de esa ciudad, con «restos de pintura roja» cuya situación no precisan sus editores (Santos, Hoces y del Hoyo 2005,161, n. $^{\circ} 80$ y 184, n. $\left.^{\circ} 106\right)$. A juzgar por la foto de una de ellas parecen distinguirse trazas de pintura por todo el campo epigráfico. También se ha preservado la coloración en un altarcito dedicado a las Duillae del Museo Arqueológico de Palencia (Del Amo y Pérez 2006, 96). Más exponentes de rubricatura se conocen en Portugal en granito o mármol, como la estela de C. Iulius Letondo de Courela (Ourique), un ara a I.O.M. de Vilariça (Vila Real), una cupa de Mértola y un pedestal con dedicación a Esculapio de Lisboa (Cardim Ribeiro (coord.) 2002).

Finalmente, en lo que respecta al contexto del pequeño monumento epigráfico que nos ocupa, hay que decir que el conjunto de enclaves arqueológicos que compone el yacimiento de Almenara de Adaja está situado a escasos $\mathrm{km}$ de Cauca a cuyo territo- rio posiblemente perteneciese. Mas cerca aún hubo un extenso núcleo de población de época imperial descubierto en prospecciones en el término de Olmedo, a escasos $8 \mathrm{~km}$ al norte de la villa (Centeno 1994, 150-157), tal vez un núcleo secundario del territorio caucense, tal vez distinto. La residencia señorial excavada de la villa de Almenara corresponde a la fase final-segunda mitad del siglo iv y siglo $\mathrm{v}$ de esta instalación. Hay evidencias de una explotación y residencia del siglo III y la primera mitad del Iv y materiales que ponen de manifiesto la ocupación del sector desde finales del siglo i (García Merino y Sánchez Simón 2004, 180-181; García Merino, e.p).

\section{BIBLIOGRAFÍA}

ABASCAL, J. M., 2002: «La Era consular hispana y el final de la práctica epigráfica pagana». Lucentum XIX-XX, 2000-2001, 269-292.

Blázquez. J. M. ${ }^{a}$, 1981: «El sincretismo en la Hispania romana entre las religiones indígenas, griega y romana, fenicia y mistéricas». La religión romana en Hispania. Madrid, 179-221.

- 1983: Primitivas religiones ibéricas II. Religiones prerromanas. Madrid.

CARdim Ribeiro, J. (coord.), 2002: Religiôes da Lusitania. Loquuntur Saxa. Catalogo de la exposición. Lisboa.

Centeno CeA, I., 1994: «Época romana». Inventario arqueológico de la provincia de Valladolid. Informe de la Campaña de 1992/1993. Inédito y depositado en la Delegación territorial de Cultura de la Junta de Castilla y León en Valladolid, 150-157.

Del Amo, M.; Pérez, F. J., 2006: Guía del Museo de Palencia. Junta de Castilla y León. Palencia.

García Merino, C.; Sánchez Simón, M., 2004: «De nuevo acerca de la villa romana de Almenara de Adaja (Valladolid). Excavaciones de 1998 a 2002». AEspA 77, 177-195.

García Merino, C. (e.p.): «Almenara de Adaja y las villas de la meseta septentrional». IV Coloquio Internacional de Arqueología en Gijón. Las villas tardorromanas en el Occidente del Imperio: Arquitectura y función (Gijón, 26-28 de Octubre de 2006).

Gimeno Pascual, H.; Stylow, A., 2004: «Epigrafía de la Beturia céltica en la Casa de Pilatos (Sevi1la)», en C. Auliard \& L. Broliou (ed.): Au jardin des Hespérides: histoire, societé et epigraphie des mondes anciennes. Melanges offerts à Alan Tranoy. Rennes, 77-85 
GonZÁlez, J., 1996: Corpus de Inscripciones latinas de Andalucía. II. Sevilla. Sevilla.

Grünhagen, W., 1977: «El monumento a Dis Pater de Munigua». Segovia y la Arqueología romana. Symposium Internacional de Arqueología romana. Bimilenario de Segovia. (Segovia, 1974) Barcelona, 201-208.

KNAPP, R. C., 1992: Latin inscriptions from Central Spain. Berkeley.

LINDER, R., 1988: «Hades-Pluto». Lexicon iconographicum mythologiae classicae. IV, 1, Zürich, 399406.

Mañanes, T.; Solana, J. M., 1999: Inscripciones de época romana de la provincia de Valladolid. Valladolid.

Molinero, A., 1971: Aportaciones de las excava- ciones y hallazgos casuales (1941-1959) al Museo arqueológico de Segovia. MJSEA, 98 (1927).

Palol, P. de; Vilella, J., 1987: Clunia II. La epigrafía de Clunia. E.A.E. 150. Madrid.

Puinius, C.: Naturalis historia. Ed. H. Rackham. Lib. XXXIII-XXXIV. Ed. Loeb. Cambridge, 1952.

Santos Yanguas, J.; Hoces de la Guardia Bermejo, A. L.; Del Hoyo, J., 2005: Epigrafía romana de Segovia y su provincia. Segovia.

Taracena Aguirre, B., 1941: Carta arqueológica de España. Soria. Madrid, 85.

Recibido el 06-03-08. Aceptado el 15-07-08. 